\title{
PLANET SHADOWS IN PROTOPLANETARY DISKS. II. OBSERVABLE SIGNATURES
}

\author{
HANNAH JANG-CONDELL ${ }^{1,2,3}$ \\ ${ }^{1}$ Department of Astronomy, University of Maryland, College Park, MD 20742, USA; hannah@astro.umd.edu \\ 2 NASA Goddard Space Flight Center, Greenbelt, MD 20771, USA \\ Received 2009 March 16; accepted 2009 May 25; published 2009 July 6
}

\begin{abstract}
We calculate simulated images of disks perturbed by embedded small planets. These $10-50 M_{\oplus}$ bodies represent the growing cores of giant planets. We examine scattered light and thermal emission from these disks over a range of wavelengths, taking into account the wavelength-dependent opacity of dust in the disk. We also examine the effect of inclination on the observed perturbations. We find that the perturbations are best observed in the visible to mid-infrared (mid-IR). Scattered light images reflect shadows produced at the surface of perturbed disks, while the infrared images follow thermal emission from the surface of the disk, showing cooled/heated material in the shadowed/brightened regions. At still longer wavelengths in the submillimeter, the perturbation fades as the disk becomes optically thin and surface features become overwhelmed by emission closer toward the midplane of the disk. With the construction of telescopes such as TMT, GMT, and ALMA due in the next decade, there is a real possibility of observing planets forming in disks in the optical and submillimeter. However, having the angular resolution to observe the features in the mid-IR will remain a challenge.
\end{abstract}

Key words: planetary systems: formation - planetary systems: protoplanetary disks - radiative transfer

\section{INTRODUCTION}

Giant planet formation remains an unsolved problem. The two leading theories are disk instability and core accretion. In disk instability, the protoplanetary disk gravitationally fragments into Jupiter-mass clumps which condense into planets. In core accretion, smaller bodies agglomerate into larger and larger bodies until they are massive enough to accrete a gaseous envelope. There are theoretical arguments for and against both scenarios, but the aim here is to identify signatures of planet formation in order to provide direct observational evidence for one scenario or the other. Simulated scattered light signatures of planet formation by disk instability have been discussed elsewhere (Jang-Condell \& Boss 2007), so the focus of this paper is on signatures of core accretion.

Giant planets forming by core accretion need to have cores of $10-20 M_{\oplus}$ to be massive enough to accrete a gaseous envelope (Hubickyj et al. 2005). For reference, Jupiter is approximately $300 M_{\oplus}$. This predicts that sizeable planet embryos form before circumstellar gas disks dissipate. These disks are typically modeled as passive accretion disks, meaning that the dominant heating source is stellar irradiation, although there still is sufficient viscous dissipation for the disk to continue accretion at a slow rate onto the star $\left(\lesssim 10^{-7} M_{\odot} \mathrm{yr}^{-1}\right)$ and for a modest amount of heating at the disk midplane. These disks are optically thick and gas-dominated. Their temperature structure is strongly dependent on heating from stellar irradiation, particularly on the angle of incidence of the starlight on the disk surface (Chiang \& Goldreich 1997; Calvet et al. 1991; D'Alessio et al. 1998, 1999, 2001).

We examine planets forming at distances of 1-8 AU from the stars. Interior to $1 \mathrm{AU}$, the innermost regions of protoplanetary disks are expected to become optically thin because of the sublimation of dust grains, creating a hot inner rim at $\sim 0.1-$ 0.5 AU (Dullemond et al. 2001; Isella \& Natta 2005; D’Alessio et al. 2006). This is supported by observations of T Tauri stars in the near-infrared (near-IR) to mid-infrared (mid-IR; Eisner

\footnotetext{
3 Michelson Fellow.
}

et al. 2009, 2005; Muzerolle et al. 2003). Although larger radii for inner holes in protoplanetary disks are observed, this is typically for higher mass Herbig Ae/Be stars, which are both hotter and more luminous than $\mathrm{T}$ Tauri stars, resulting in the evaporation of dust grains at larger distances from the star. The typical inner disk radius for T Tauri stars is below $0.5 \mathrm{AU}$, which is the minimum disk distance modeled in this study. For this reason, the emission from the inner disk rim can be treated as a separate problem from the study presented in this paper.

Other work on simulated images of growing planets focuses on Jovian-mass planets rapidly accreting material from their surroundings disks. These planets are large enough to create large-scale structures in disks such as gaps or spiral density waves, and the planets themselves are observable because they are heated by accretion (e.g., Wolf 2008; Klahr \& Kley 2006). Planet formation has already completed and it is difficult to determine the mechanism by which it occurred. By contrast, the study presented in this paper focuses on planets which are too small to open gaps or accrete significant amounts of gas $\left(10-50 M_{\oplus}\right)$.

The planet-disk models adopted here are presented in JangCondell (2008) and summarized in this section. The planet is predicted to gravitationally compress the disk in the vertical direction, creating a shadow paired with a bright spot, leading to temperature variations. We calculate both scattered light and thermal emission from the perturbed disk for planets of mass 10-50 $M_{\oplus}$ at distances of 1-8 AU from their host stars.

In Section 2, we describe the procedure we use for modeling radiative transfer in the disk. In Section 3, we show the results of the modeling for the full suite of planet-disk models over a range of wavelengths. In Section 4, we discuss the potential observability of the planet perturbations we have modeled. In Section 5 we present our conclusions.

\section{MODEL DESCRIPTION}

\subsection{Disk Model: Thermal Structure}

The model for thermal perturbations in the vicinity of an embedded protoplanet are presented in Jang-Condell (2008). 
The stellar parameters are mass $M_{*}=1 M_{\odot}$, radius $R_{*}=$ $2.6 R_{\odot}$, and effective temperature $T_{\text {eff }}=4280 \mathrm{~K}$. The radius and effective temperature correspond to a stellar age of $1 \mathrm{Myr}$ (Siess et al. 2000). We assume a viscosity parameter of $\alpha=0.01$ and accretion rate $\dot{M}=10^{-8} M_{\odot} \mathrm{yr}^{-1}$, which are typical of T Tauri stars (Hartmann et al. 1998; D’Alessio et al. 1998).

The model for the disk is based on an accretion disk model with viscosity parametrized by a dimensionless constant $\alpha$, so that the viscosity is $v=\alpha c_{s} H$ where $c_{s} \equiv \sqrt{k T_{0} / \bar{m}}$ is the thermal sound speed at the midplane of the disk and $H \equiv c_{s} / \Omega$ is the pressure scale height (Shakura \& Sunyaev 1973). Here, $k$ is the Boltzmann constant, $T_{0}$ is the midplane temperature, $\bar{m}$ is the mean molecular weight of molecular hydrogen, and $\Omega$ is the Keplerian angular velocity. For the low accretion rate adopted and the distances in the disk in which we are interested, stellar irradiation heating dominates, though we include viscous heating for completeness.

The amount of heating from stellar irradiation at the surface depends on the angle of incidence of the stellar rays. Based on an analytic solution to the one-dimensional plane-parallel case, we calculate surface heating semianalytically in a $1+2 \mathrm{D}$ manner, where we numerically integrate heating contributions over the shape of the disk surface. We include the differential rotation of the disk by calculating the rate of radiative cooling and heating as disk material enters or exits the shadowed or brightened regions.

We assume vertical hydrostatic equilibrium in the disk, including contributions to the vertical gravity from both the star and the planet. We treat the planet embedded in the disk as a point mass, whose gravitational potential compresses the disk in the vertical direction, creating a dimple at the surface. The dimple at the surface results in a shadow at the planet's position relative to the unperturbed disk, while the outer edge is brightened.

The radiative transfer calculations rely on a separation of the radiation into two regimes: the short wavelength spectrum of the stellar emission, and the long wavelength spectrum of the thermal emission of the disk material. We use the opacities from D'Alessio et al. (2001) using a dust model with parameters $a_{\max }=1 \mathrm{~mm}, T=300 \mathrm{~K}$, and $p=3.5$, assuming that the dust opacities are constant throughout the disk. The values for the opacities (in $\mathrm{cm}^{2} \mathrm{~g}^{-1}$ ) are as follows: the Rosseland mean opacity is $\chi_{R}=1.91$, the Planck mean opacity integrated over the disk spectrum $(300 \mathrm{~K})$ is $\kappa_{P}=0.992$, and the Planck mean opacities integrated over the stellar spectrum $(4000 \mathrm{~K})$ are $\kappa_{P}^{*}=1.31$ for absorption alone and $\chi_{P}^{*}=5.86$ for absorption plus scattering. The absorption fraction is then $\alpha_{\mathrm{abs}}=\kappa_{P}^{*} / \chi_{P}^{*}$, while the scattered fraction is $\sigma=1-\alpha_{\text {abs. }}$. The Rosseland mean opacity is used to calculate the photosphere of the disk, and $\chi_{P}^{*}$ is used to calculate the surface of the disk. We assume that the dust is well mixed with the gas and constant throughout the disk.

The initial conditions vary slightly in the overlap regions between simulations as the size and position of the simulation box vary. The sizes of the boxes are scaled to the Hill radius,

$$
r_{\text {Hill }}=\left(\frac{m_{p}}{3 M_{*}}\right)^{1 / 3} a \text {. }
$$

Thus, the boundary conditions depend on the mass and the distance of the planet. In order to make a fair comparison between the various planet models, we assemble a composite disk from the initial conditions of the largest boxes at each distance from the star. We interpolate the densities and temperatures

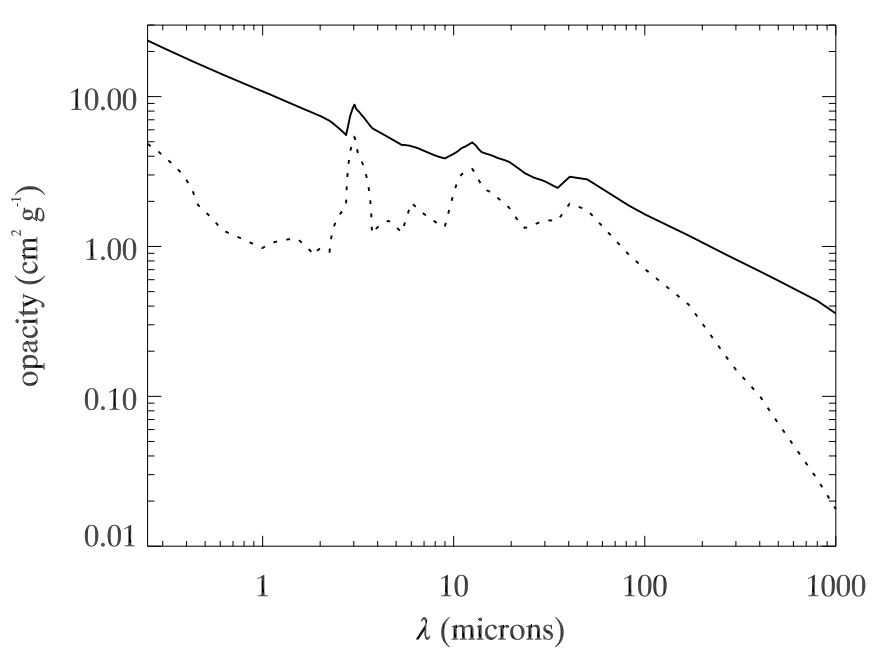

Figure 1. Wavelength-dependent opacities for the dust model adopted for calculating simulated images. Dotted line: absorption only. Solid line: the total extinction (absorption+scattering).

smoothly across the overlap regions. The magnitude of the variations in temperature and density should roughly scale, so we normalize the temperatures and densities of each planet-disk model to this composite set of initial conditions.

\subsection{Opacities}

In order to calculate simulated images of the disk, we need a good model for the opacities. Although mean opacities were good enough for calculating the thermal structure of the disk, to calculate images of the disk, we need the frequency-dependent absorption and total extinction coefficients for the dust model.

The opacities were calculated using a Mie scattering code developed by Pollack \& Cuzzi (1980). The composition of the dust is that used in Pollack et al. (1994), consisting of water, troilite, astronomical silicates, and organics. We adopt a size distribution for the dust of $n(a) \propto a^{-3.5}$ where $a$ is the radius of the grain, with maximum and minimum radii of $1 \mathrm{~mm}$ and $0.005 \mu \mathrm{m}$, respectively. The large maximum grain size represents coagulation of grains in protoplanetary disks.

In Figure 1, we show the absorption (dotted line) and total extinction (solid line) coefficients in units of $\mathrm{cm}^{2} \mathrm{~g}^{-1}$ versus wavelength for our adopted dust model. The total extinction includes both scattering and absorption. If $\kappa_{v}$ and $\chi_{v}$ are the frequency-dependent absorption and extinction coefficients, respectively, then the albedo is the ratio of scattering to total extinction, so $\omega_{\nu}=1-\kappa_{\nu} / \chi_{\nu}$. Since we assume that the dust is well mixed, the dust density simply scales with the gas density.

\subsection{Scattered Light Imaging}

We calculate scattered light from the disk assuming that it is face on and that stellar irradiation is primarily scattered from part of the disk that is optically thin to stellar irradiation. The optical depth to stellar light at a frequency $v$ is

$$
\tau_{v, *}=\int_{\ell} \chi_{\nu} \rho d l,
$$

where $\chi_{v}$ is the extinction coefficient, $\rho$ is the density of the disk, and $\ell$ is the path between the star and the point in the disk. From this point on, the dependence on frequency, $v$, will be implicit and we will omit the subscripts. We define the surface of the disk, $z_{s}(r)$ to be where $\tau_{*}=2 / 3$. The cosine of the angle 

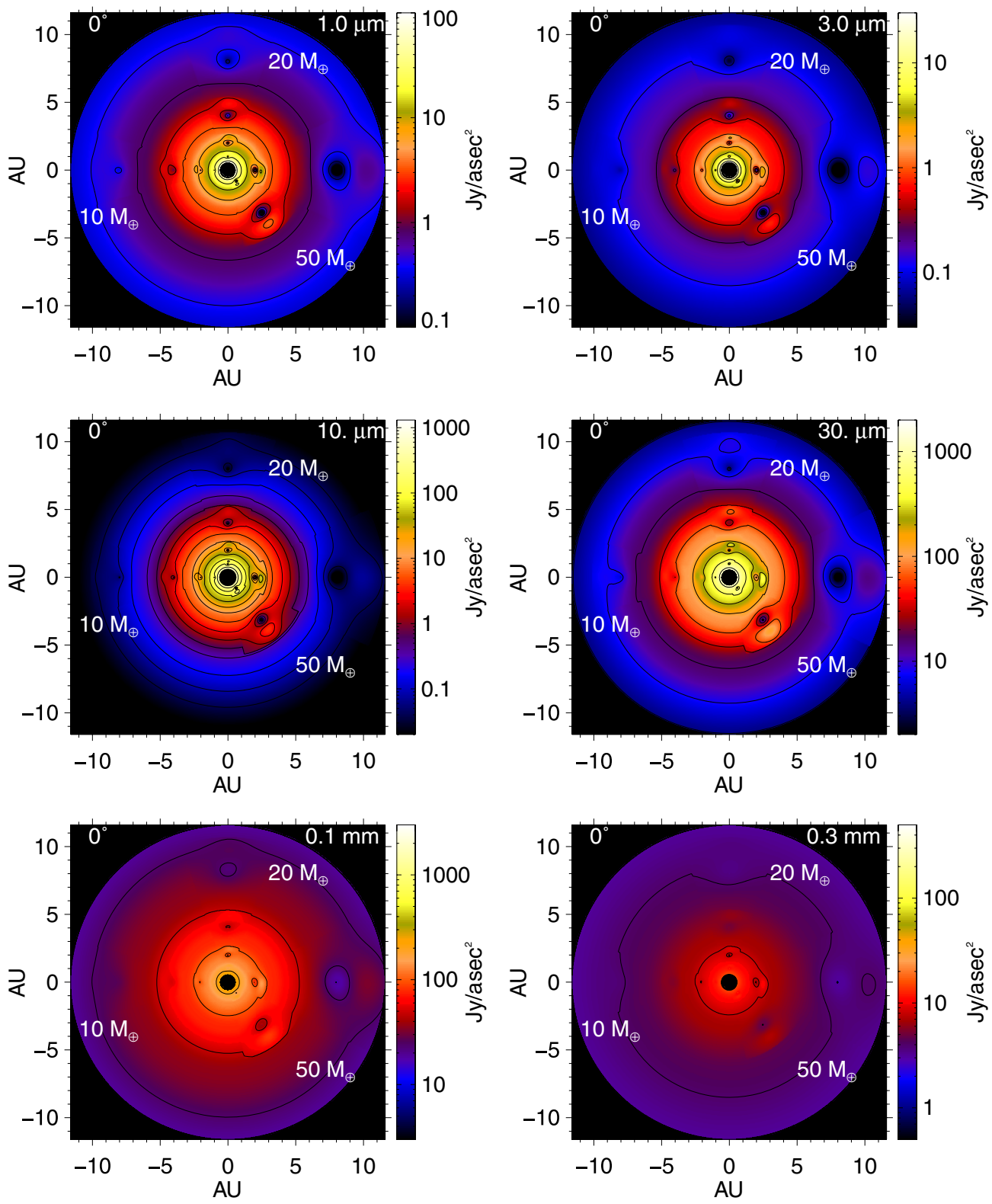

Figure 2. Planets of varying masses embedded in a face-on disk at 1, 2, 4, and $8 \mathrm{AU}$ seen at varying wavelengths. From left to right, top to bottom: 1, 3, 10, 30, 100, and $300 \mu \mathrm{m}$. Planets at the 9 o'clock position are $10 M_{\oplus}$, planets at the 12 o'clock position are $20 M_{\oplus}$, and planets at the $3-5$ o'clock positions are $50 M_{\oplus}$.

of incidence at radius $r$ is

$$
\mu(r)=\frac{\left(z_{s}^{\prime}-z_{s} / r\right)}{\left[\left(z_{s}^{\prime}\right)^{2}+1\right]^{1 / 2}\left(1+z_{s}^{2} / r^{2}\right)^{1 / 2}}+\frac{4 R_{*}}{3 \pi\left(r^{2}+z_{s}^{2}\right)^{1 / 2}} .
$$

The first term is a purely geometric term, and the second term accounts for the finite size of the star, in contrast to illumination by a point source. The slope of the surface is defined as $z_{s}^{\prime}=d z_{s} / d r$. We use a log-log interpolation to calculate both $z_{s}$ and $z_{s}^{\prime}$ as a function of $r$.

Assuming isotropic scattering, the brightness of disk as a function of frequency, $I_{v}^{s}$, can be calculated by integrating the following equation from D'Alessio et al. (1999):

$$
\frac{d I^{s}}{d Z}=-\frac{(\chi-\kappa) R_{*}^{2}}{4 l^{2}} B\left(T_{*}\right) \exp \left[-\tau_{*}-\tau_{\mathrm{obs}}(Z)\right],
$$

where $l$ is the distance to the star, $B\left(T_{*}\right)$ is the Planck function at the effective temperature of the star, and $\tau_{\mathrm{obs}}$ is the optical depth to the observer, $\tau_{\mathrm{obs}}=\int_{\mathrm{obs}} \chi_{\nu} \rho d l$, integrated along the line of sight from the observer to the disk. For a face-on disk, this is integrated along the vertical axis, so $Z=z$.

At low optical depths, $\rho$ is small, so contributions to $I^{s}$ are small. At high optical depths, the exponential term goes to 0 , so those contributions are also small. Only the regions close to the surface contribute significantly to the scattered light intensity, so we assume $l \approx \sqrt{r^{2}+z_{s}^{2}}$. We assume that the disk is locally plane-parallel at the surface, so that if $i$ is the angle between the line of sight to the observer and normal to the surface, $\tau_{*}=\cos i \tau_{\mathrm{obs}}(z) / \mu$. Note that $i$ is not the inclination of the disk, but rather the angle with respect to the disk surface, which is not generally parallel to the ecliptic. Then Equation (4) becomes

$$
\frac{1}{\chi \rho} \frac{d I^{s}}{d z}=\frac{d I^{s}}{d \tau}=\frac{\omega R_{*}^{2}}{4\left(r^{2}+z_{s}^{2}\right)} B\left(T_{*}\right) \exp [-(1+\cos i / \mu) \tau] .
$$




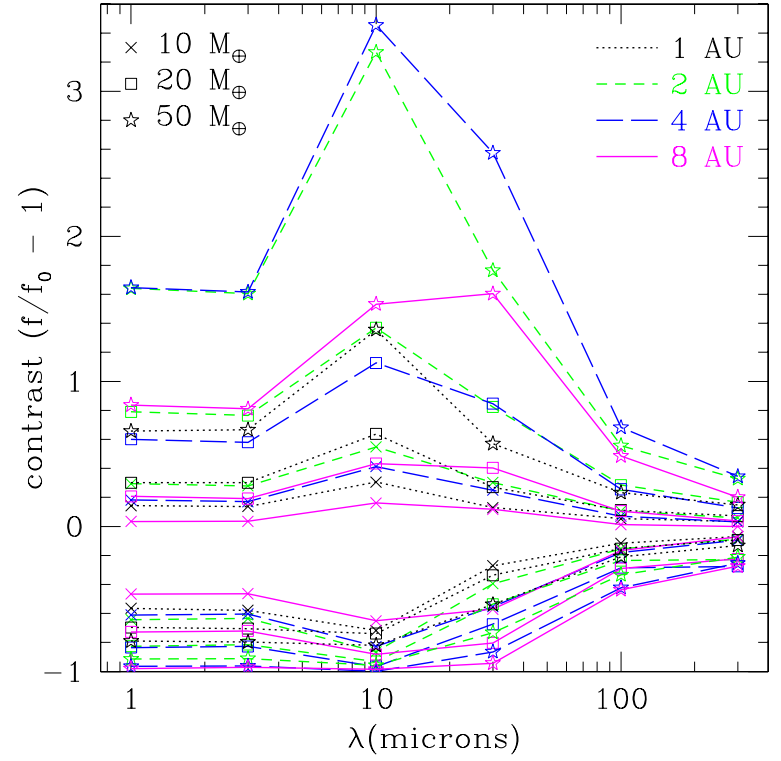

Figure 3. Minimum/Maximum contrast vs. wavelength. Orbital distances of $1,2,4$, and $8 \mathrm{AU}$ are represented by black dotted, green short-dashed, blue long-dashed, and solid magenta lines, respectively. Planet masses are indicated by crosses, squares, and stars for 10,20 , and $50 M_{\oplus}$, respectively.

Integrating from $\tau=0 \rightarrow \infty$,

$$
I^{\text {scatt }}=\frac{\omega \mu R_{*}^{2} B\left(T_{*}\right)}{4\left(r^{2}+z_{s}^{2}\right)(\mu+\cos i)} .
$$

\subsection{Thermal Emission}

At longer wavelengths, thermal emission from the disk material becomes important. The thermal emission from a disk is calculated following D'Alessio et al. (1999), by integrating the equation

$$
\frac{d I^{t}}{d Z}=\kappa \rho B\left(T_{d}\right) \exp \left(-\tau_{\mathrm{obs}}\right)
$$

along the line of sight, where $T_{d}$ is the local disk temperature and $B\left(T_{d}\right)$ is the thermal emission at that temperature. The thermal emission and scattered light are summed to give the total disk surface brightness

$$
I^{\mathrm{disk}}=I^{s}+I^{t} .
$$

\section{RESULTS}

In Figures 2-10, we show the results of the simulated images of planets in disks from 1 to $300 \mu \mathrm{m}$. Face-on disks $\left(0^{\circ}\right.$ inclination) with planets are displayed in Figure 2. Each disk image shows a different wavelength, with 10,20 , and $50 M_{\oplus}$ planets embedded at $1,2,4$, and $8 \mathrm{AU}$, respectively. The $10 M_{\oplus}$ planets are at the $9 \mathrm{o}$ 'clock position and the $20 M_{\oplus}$ planets are the 12 o'clock position. The $50 M_{\oplus}$ planets are in the lower right quadrant of the disk. They are offset from each other because their simulation boxes overlap slightly in the radial direction. In subsequent figures, we allow the boxes to overlap because the perturbation inward of the planet is minimal.

The shadows are roughly centered at the planet's position in radius and azimuth, and brightened spots appear on the far side of the dimple. We define the contrast to be $c=f / f_{0}-1$ where $f_{0}$ is the radially dependent unperturbed disk brightness and $f$ is the disk brightness in the presence of the planet. The depths of the shadows/bright spots regions in the face-on disk can

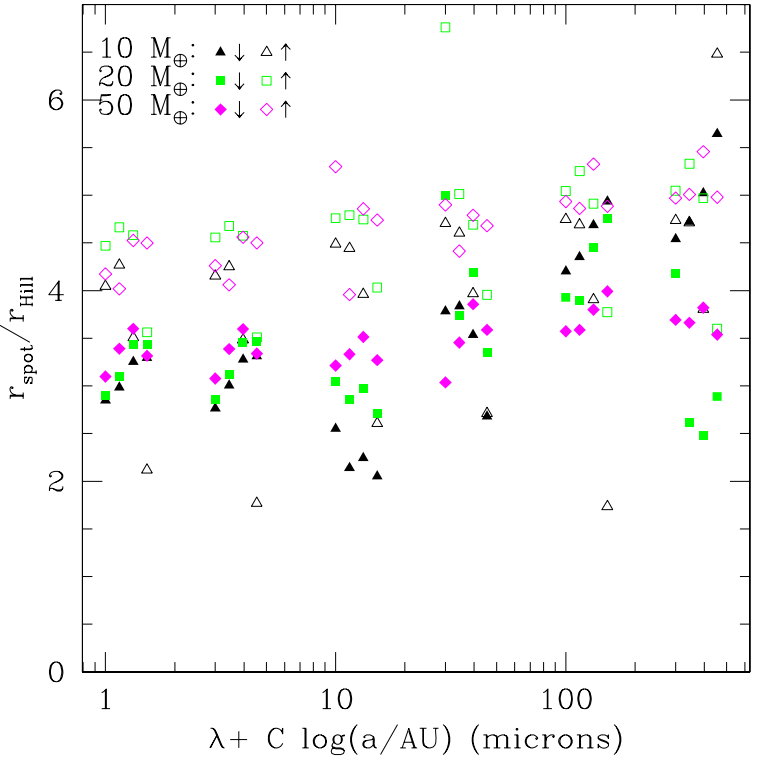

Figure 4. Equivalent radii $r_{\text {spot }}$ of shadowed/brightened regions, scaled to the Hill radius $r_{\text {Hill }}$, vs. wavelength. The points have been horizontally shifted to distinguish between orbital distances, but the wavelengths correspond to 1,3 , $10,30,100$, and $300 \mu \mathrm{m}$ exactly. Black triangles, green squares, and magenta diamonds indicate planet masses of 10, 20, and $50 M_{\oplus}$, respectively. Filled symbols indicate shadows and open symbols indicate bright spots.

then be characterized by the minimum/maximum value of the contrast. The sizes of the shadows/bright spots are characterized by the area enclosed by a contour traced at half the minimum/ maximum contrast. These values are tabulated in Columns 5, 6,8 , and 9 of Table 1 by planet mass (Column 1), orbital distance (Column 2), and wavelength of observation (Column 4). The "spot radius" is the equivalent radius of the half- $\min / \mathrm{max}$ areas $A$,

$$
r_{\mathrm{spot}}=\sqrt{\frac{A}{\pi}}
$$

In Figure 3, we plot the maximum/minimum values of the contrast versus wavelength, with line type and color indicating orbital distance and symbols indicating planet mass. The contrasts generally increase with mass, while the dependence on distance in more complicated. The wavelength dependence will be discussed in further detail below.

Figure 4 shows the dependence of $r_{\text {spot }} / r_{\text {Hill }}$ with wavelength, with symbol shape and color indicating mass, and horizontal offset indicating distance. The clustering of the points indicates that $r_{\text {spot }}$ scales roughly with $r_{\text {Hill }}$, with brightened spots being generally larger than shadows. In other words, the size of the shadow scales roughly with the distance from the star and the cube root of the planet mass.

In Figures 5, 6, 7, 8, 9, and 10 we show the effect of inclination on the appearance of the perturbed disk at wavelengths of 1,3 , $10,30,100$, and $300 \mu \mathrm{m}$, respectively. Each figure shows, from left to right, an angle of inclination of $30^{\circ}, 45^{\circ}$, and $60^{\circ}$. From top to bottom, the planet mass is 10,20 , and $50 M_{\oplus}$. The inclination axis is always pointing upward, so that the upper part of the disk is further from the observer than the bottom part. In each disk model, planets are inserted at 1,2, 4, and $8 \mathrm{AU}$, along each minor and major axis to demonstrate how the phase of the planet affects the appearance of the perturbation. 

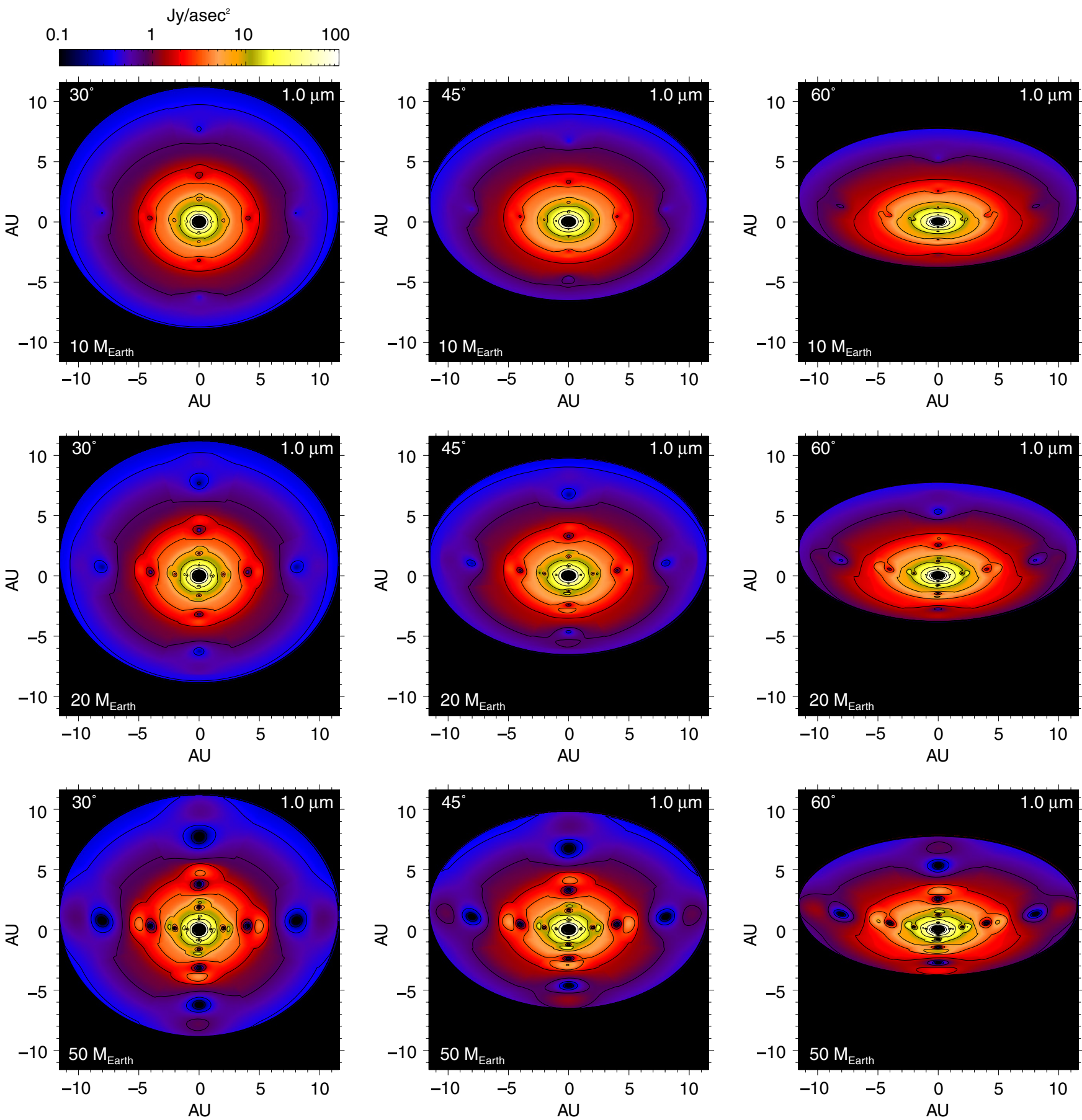

Figure 5. Simulated images of inclined disks perturbed by embedded planets at $1 \mu \mathrm{m}$. The stellar brightness at $1 \mu \mathrm{m}$ is $1.54 \mathrm{Jy}$ at a distance of $100 \mathrm{pc}$. Each image shows how a planet of a given mass will perturb the appearance of the disk at $1,2,4$, and $8 \mathrm{AU}$, and at phases $0, \pi / 2, \pi$, and $3 \pi .2$. From left to right, the disks are inclined at 30,45 , and $60^{\circ}$. The planet masses from top to bottom are 10,20 , and $50 M_{\oplus}$.

\subsection{Scattered Light}

In the optical to near-IR ( 1 and $3 \mu \mathrm{m}$, upper plots in Figure 2), the image of the disk is predominantly from scattered light. These images trace the contours of the surface of the disk. The shadowing and illumination are clearly visible. For reference, the stellar brightness is $1.54(d / 100)^{-2} \mathrm{Jy}$ at $1 \mu \mathrm{m}$, and $0.77(d / 100)^{-2} \mathrm{Jy}$ at $3 \mu \mathrm{m}$, where $d$ is the distance to the star. At these wavelengths, the brightness of the star overwhelms any emission from the disk, so very good starlight suppression, i.e., coronography, is necessary to resolve any features in the disk. Coronography is also a good way to suppress emission from hot inner walls of the disk, since this may be a significant source of near-IR flux in the inner $0.5 \mathrm{AU}$ of the disk.

The shadow contrast is generally deeper than the bright contrast because it is easier to create a depression than it is to puff up disk material due to heating, especially in the surface layers of the disk where the density is very low. As seen in JangCondell (2008), the heating from illumination is greater interior to $8 \mathrm{AU}$ because the densities are higher in the inner regions. The exception to this is for the largest planet at 2-4 AU, where 

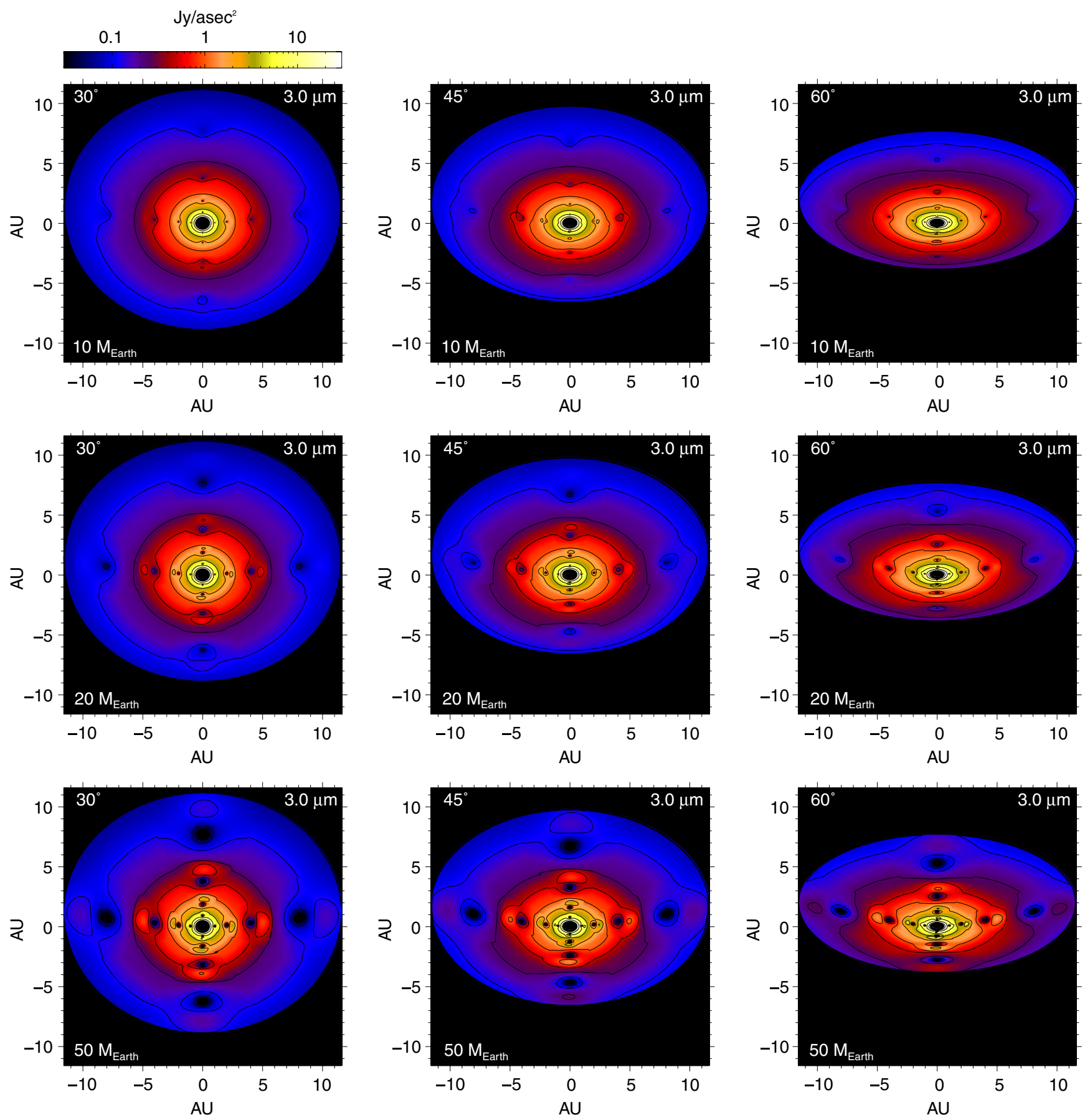

Figure 6. Simulated images of inclined disks perturbed by embedded planets at $3 \mu \mathrm{m}$. The stellar brightness at $3 \mu \mathrm{m}$ is $0.77 \mathrm{Jy}$ at a distance of $100 \mathrm{pc}$. See Figure 5 for description.

the bright contrast rises above 1 , whereas the shadow contrast has a floor at -1 . On the other hand, the area of the brightened regions are generally larger than the shadowed region, except for the smallest planet at $8 \mathrm{AU}$, where the amount of brightening is very small.

The effect of inclination on planet dimples can be seen in Figures 5 and 6 . One general effect is that as the angle of inclination increases, the brightness of the disk also increases. Examining Equation (6), we see that as $i$, the angle of observation with respect to the surface normal, increases, $\cos i$ decreases, increasing the overall value of $I^{s}$. The interpretation for this is that the observer is viewing more scatterers along the line of sight through the disk, thus the disk appears brighter. Since the disk images are tipped up so that the bottom half is closer to the observer, the value of $i$ is greater, so the bottom halves appear brighter. The planet perturbation on top of this general effect causes the rebrightened region of the disk to appear brighter still when it is on the near side.

\subsection{Mid-IR: Thermal Emission from the Surface}

The mid-IR (10 and $30 \mu \mathrm{m}$, center plots in Figure 2) probes thermal emission from the surface layers of the disk. At these wavelengths, the observed features are caused by temperature perturbations resulting from cooling in the shadowed regions and heating in the illuminated regions. The size and contrast of the perturbations show the same variation with planet size and 

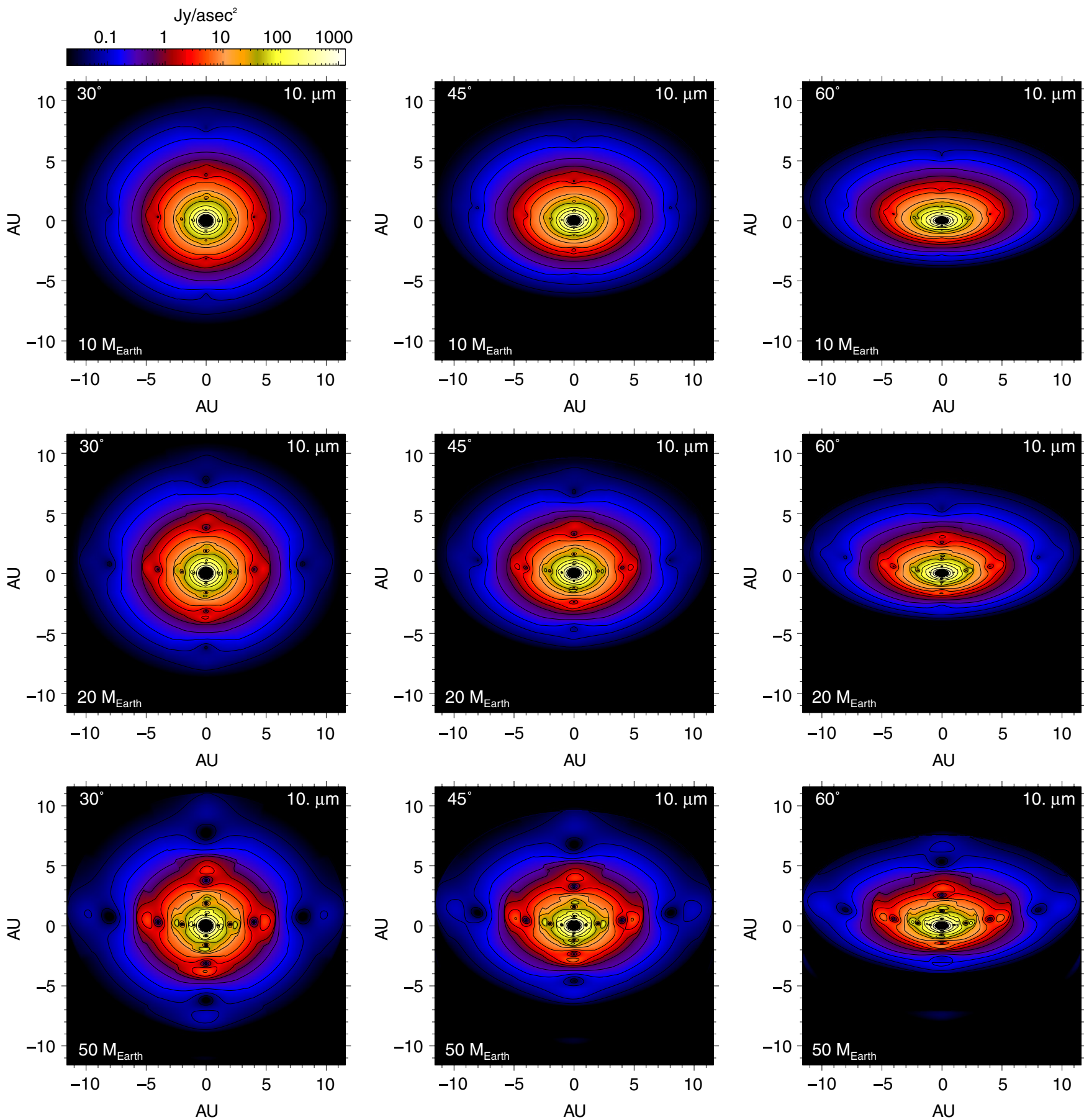

Figure 7. Simulated images of inclined disks perturbed by embedded planets at $10 \mu \mathrm{m}$. The stellar brightness at $10 \mu \mathrm{m}$ is $0.107 \mathrm{Jy}$ at a distance of $100 \mathrm{pc}$. See Figure 5 for description.

distance as seen in the scattered light images. Again, the shadows appear deeper than the brightened spots, except when the bright contrast $\gtrsim 1.8$ because of the natural floor in the shadow contrast at -1 . The stellar brightness is $0.107(d / 100)^{-2} \mathrm{Jy}$ at $10 \mu \mathrm{m}$, and $1.34 \times 10^{-2}(d / 100)^{-2} \mathrm{Jy}$ at $30 \mu \mathrm{m}$.

The greatest amount of contrast occurs at $10 \mu \mathrm{m}$. The $10 \mu \mathrm{m}$ image also shows the most radial contrast in the disk overall because the outer regions of the disk are too cold to emit effectively at this wavelength: $10 \mu \mathrm{m}$ is shortward of the exponential cutoff in thermal emission in the outer disk. The steep background radial gradient of the unperturbed disk itself will make it difficult to isolate perturbations from embedded planets.
In the inclined disks (Figures 7 and 8), there appears to be a brightening associated with higher inclinations, similar to the scattered light images, but of lesser magnitude. This brightening is caused by an optical depth effect, because as more heated or cooled material aligns along the line of sight, the stronger or weaker the thermal emission becomes.

\subsection{Far-IR to Radio: Thermal Emission from the Midplane}

At submillimeter wavelengths, the stellar brightness is negligible compared with the thermal emission from the disk. In this regime the perturbations induced by the embedded planets are hardly visible $(0.1 \mathrm{~mm}$ and $0.3 \mathrm{~mm}$, lower plots in Figure 2). The 

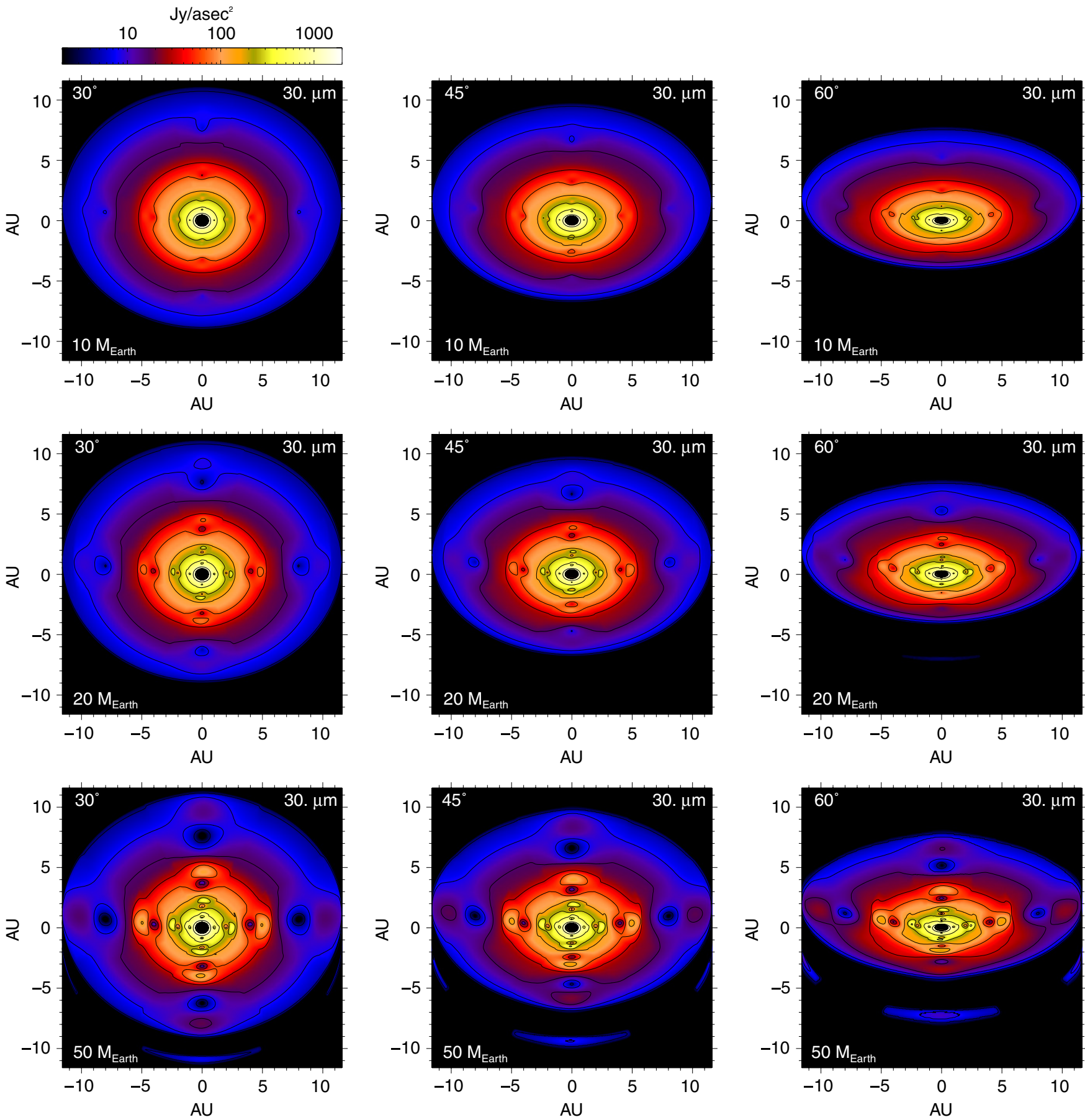

Figure 8. Simulated images of inclined disks perturbed by embedded planets at $30 \mu \mathrm{m}$. See Figure 5 for description.

contrast is modest compared to shorter wavelengths. The scatter in $r_{\text {spot }}$ at $300 \mu \mathrm{m}$ in Figure 4 is largely because the contrast is so modest that the half-min/max contours are hard to constrain.

The low contrast arises because the thermal structure of the disk is affected primarily in the upper layers of the disk. As the disk becomes optically thick to its own thermal radiation, the effects of small perturbations at the surface become washed out. The contrast between the inner and outer disk also becomes less pronounced because these wavelengths are in the RayleighJeans regime of the blackbody spectra.

However, other effects not modeled in this study may very well create observable signatures. In particular, dynamics within the Hill sphere is not properly included. Circumplanetary disk formation and accretion onto the forming planet will create a density enhancement and heating at the midplane, which will increase the thermal emission in this region, particularly at wavelengths where the disk becomes optically thin. Better models need to be carried out to properly model planet signatures in the radio, particularly to determine whether small planets create shadows versus brightenings.

\section{DISCUSSION: OBSERVABILITY}

In order to observe the phenomena modeled in this paper, we need to look to nearby star-forming regions to find sufficiently young protoplanetary disks. The Taurus-Auriga star forming is 

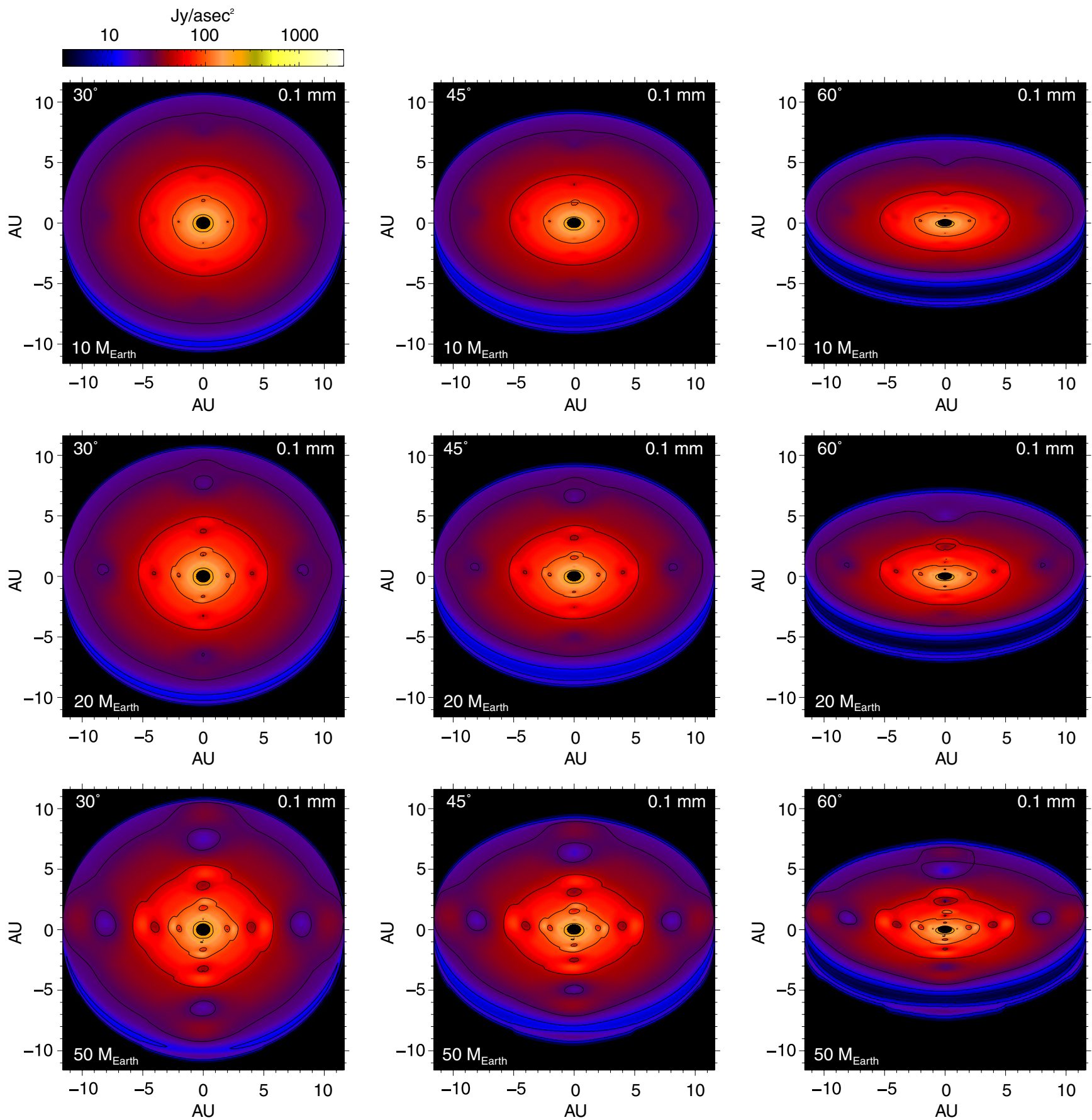

Figure 9. Simulated images of inclined disks perturbed by embedded planets at $0.1 \mathrm{~mm}$. See Figure 5 for description.

one of the nearest, at about $140 \mathrm{pc}$ (Kenyon et al. 1994). At this distance, a 1 AU perturbation subtends 0.'007.

The best wavelengths for observing planet shadows as modeled in this paper are in the visible to mid-IR. Scattered light images are sensitive to small perturbations in density at the surface of the disk. However, this is both an advantage and disadvantage. Small variations such as those caused by small planets can cause large shadowing effects. On the other hand, density variations might be caused by other processes, such as outflows, disk turbulence, and dust clumpiness, just to name a few. Differentiating planet-induced shadows from those caused by other processes may prove to be difficult.

At $1 \mu \mathrm{m}$, a diffraction limit of $\lambda / D=7$ mas requires a telescope diameter of $29 \mathrm{~m}$. This is larger than any current ground-based telescope or planned space telescope. However, with the development of large segmented-mirror telescopes such as the Giant Magellan Telescope (GMT) ${ }^{4}$ and the Thirty Meter Telescope (TMT) ${ }^{5}$ these surface shadows may be resolvable in the near future. However, scattered light observations of the planet perturbations require very good suppression of the starlight at small inner working angles.

For mas resolution at longer wavelengths, single-dish telescopes are increasingly infeasible, but interferometry is a promising alternative. The Center of High Angular Resolution Astronomy (CHARA) Array ${ }^{6}$ at Mt. Wilson Observatory

\footnotetext{
4 http://www.gmto.org/

5 http://www.tmt.org/

6 http://www.chara.gsu.edu/CHARA/index.php
} 

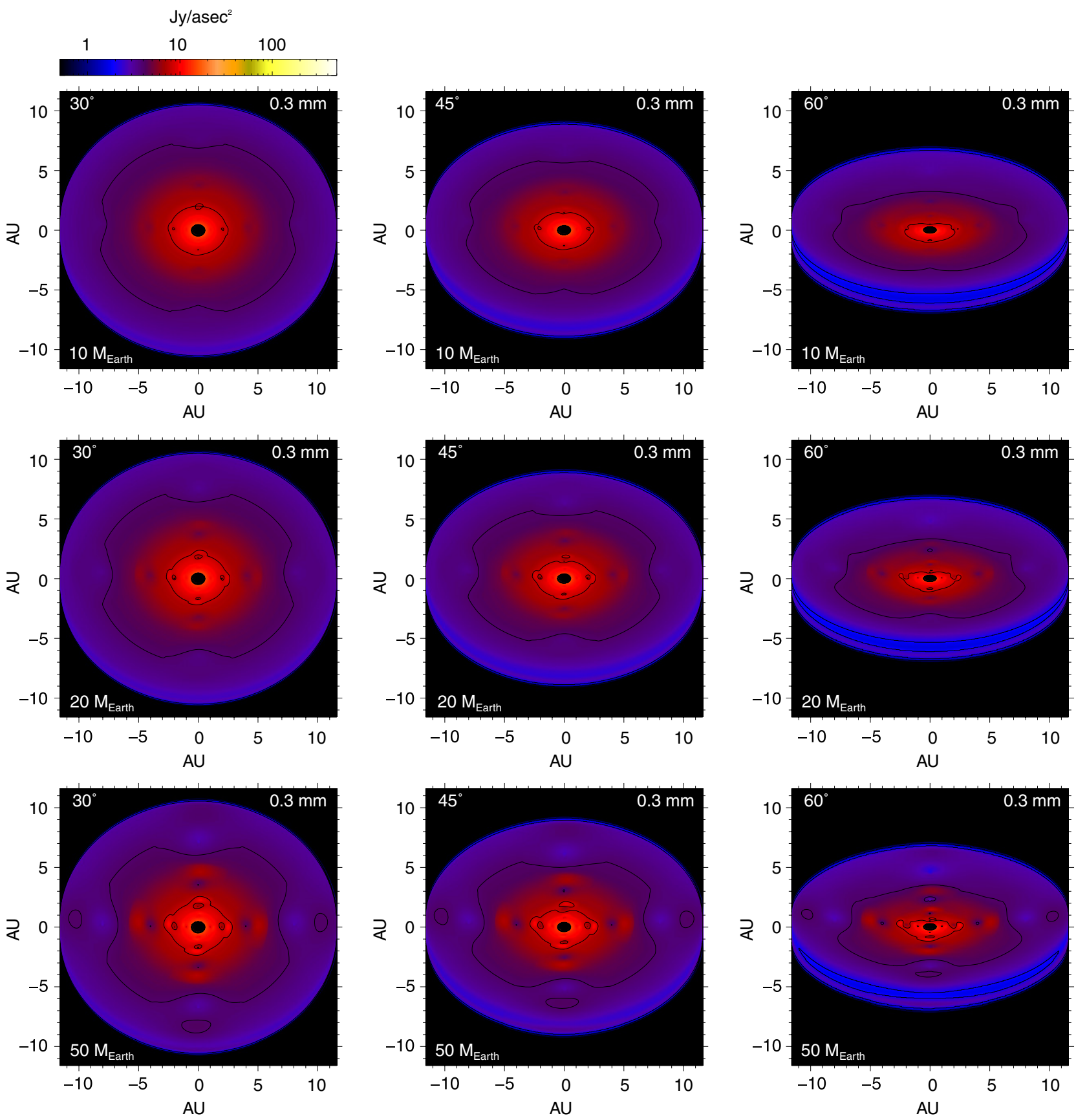

Figure 10. Simulated images of inclined disks perturbed by embedded planets at $0.3 \mathrm{~mm}$. See Figure 5 for description.

has baselines of up to $330 \mathrm{~m}$ and operates in the optical and $2.0-2.5 \mu \mathrm{m}$ range, allowing for sub-mas resolution. The Very Large Telescope Interferometer (VLTI) ${ }^{7}$ can achieve up to 2 mas resolution in $J, H$ and $K$ bands (1-2.4 $\mu \mathrm{m})$ with the AMBER instrument. VLTI-MIDI is a two-way beam combiner at $N$ band $(8-13 \mu \mathrm{m})$, for an angular resolution of about 10 mas. A nextgeneration mid-IR instrument for VLTI, MATISSE, ${ }^{8}$ is also under development for $L, N, M$, and $Q$ bands (3-25 $\mu \mathrm{m}$ ). The challenge for these instruments is covering sufficient $u-v$ space to build up an image, achieving sufficient dynamic resolution so that the star does not overwhelm the disk emission, and having enough sensitivity to detect the planet shadows.

\footnotetext{
7 http://www.eso.org/sci/facilities/paranal/telescopes/vlti/index.html 8 http://www.oca.eu/matisse/
}

Mid-IR wavelengths, which probe temperature perturbations caused by shadowing and illumination at the surface, are better for identifying planet perturbations. This is because shadows and brightenings caused by transitory phenomena will not persist as long those caused by an embedded planet, and will not result in strong cooling and heating in the surface layers. Although the shadow contrast is good at $10 \mu \mathrm{m}$, the dynamic range between the inner and outer disk is quite high, over 4 orders of magnitude from 0.5 to $10 \mathrm{AU}$. Dynamic range is less of an issue at $30 \mu \mathrm{m}$. The $30 \mu \mathrm{m}$ images shown in Figures 2 and 8 are very similar to the $1 \mu \mathrm{m}$ images in Figures 2 and 5: these two different wavelengths trace the planet perturbations equally well.

A resolving power of 7 mas at $30 \mu \mathrm{m}$ requires first getting above the Earth's atmosphere, then having a baseline of $870 \mathrm{~m}$ : this would almost certainly require a formation-flying space 
Table 1

Contrast and Size of Shadowed and Brightened Regions

\begin{tabular}{|c|c|c|c|c|c|c|c|c|c|}
\hline $\begin{array}{l}m_{p} \\
\left(M_{\oplus}\right)\end{array}$ & $\begin{array}{c}a \\
(\mathrm{AU})\end{array}$ & $\begin{array}{l}r_{\text {Hill }} \\
(\mathrm{AU})\end{array}$ & $\begin{array}{c}\lambda \\
(\mu \mathrm{m})\end{array}$ & $\begin{array}{l}\text { Shadow } \\
\text { Contrast }\end{array}$ & $\begin{array}{c}\text { Area } \\
\left(\mathrm{AU}^{2}\right)\end{array}$ & $\frac{r_{\text {spot }}}{r_{\text {Hill }}}$ & $\begin{array}{c}\text { Bright } \\
\text { Contrast }\end{array}$ & $\begin{array}{c}\text { Area } \\
\left(\mathrm{AU}^{2}\right)\end{array}$ & $\frac{r_{\text {spot }}}{r_{\text {Hill }}}$ \\
\hline 10 & 1 & 0.0216 & 1 & -0.564 & 0.0118 & 5.70 & 0.143 & 0.0239 & 8.09 \\
\hline 10 & 1 & 0.0216 & 3 & -0.578 & 0.0111 & 5.53 & 0.138 & 0.0252 & 8.31 \\
\hline 10 & 1 & 0.0216 & 10 & -0.717 & 0.00950 & 5.10 & 0.306 & 0.0294 & 8.98 \\
\hline 10 & 1 & 0.0216 & 30 & -0.270 & 0.0209 & 7.56 & 0.129 & 0.0323 & 9.41 \\
\hline 10 & 1 & 0.0216 & 100 & -0.116 & 0.0258 & 8.41 & 0.0539 & 0.0329 & 9.49 \\
\hline 10 & 1 & 0.0216 & 300 & -0.0687 & 0.0301 & 9.08 & 0.0333 & 0.0327 & 9.47 \\
\hline 10 & 2 & 0.0431 & 1 & -0.643 & 0.0519 & 5.97 & 0.295 & 0.106 & 8.54 \\
\hline 10 & 2 & 0.0431 & 3 & -0.634 & 0.0527 & 6.01 & 0.280 & 0.105 & 8.50 \\
\hline 10 & 2 & 0.0431 & 10 & -0.864 & 0.0267 & 4.28 & 0.548 & 0.115 & 8.88 \\
\hline 10 & 2 & 0.0431 & 30 & -0.395 & 0.0858 & 7.67 & 0.303 & 0.124 & 9.21 \\
\hline 10 & 2 & 0.0431 & 100 & -0.154 & 0.111 & 8.71 & 0.107 & 0.128 & 9.38 \\
\hline 10 & 2 & 0.0431 & 300 & -0.0886 & 0.130 & 9.45 & 0.0601 & 0.130 & 9.43 \\
\hline 10 & 4 & 0.0862 & 1 & -0.610 & 0.247 & 6.50 & 0.182 & 0.287 & 7.02 \\
\hline 10 & 4 & 0.0862 & 3 & -0.604 & 0.251 & 6.56 & 0.171 & 0.283 & 6.97 \\
\hline 10 & 4 & 0.0862 & 10 & -0.827 & 0.118 & 4.49 & 0.413 & 0.366 & 7.92 \\
\hline 10 & 4 & 0.0862 & 30 & -0.553 & 0.292 & 7.07 & 0.250 & 0.368 & 7.94 \\
\hline 10 & 4 & 0.0862 & 100 & -0.178 & 0.513 & 9.38 & 0.0709 & 0.356 & 7.81 \\
\hline 10 & 4 & 0.0862 & 300 & -0.0953 & 0.589 & 10.0 & 0.0320 & 0.338 & 7.61 \\
\hline 10 & 8 & 0.172 & 1 & -0.466 & 1.01 & 6.59 & 0.0346 & 0.420 & 4.24 \\
\hline 10 & 8 & 0.172 & 3 & -0.464 & 1.02 & 6.62 & 0.0352 & 0.292 & 3.54 \\
\hline 10 & 8 & 0.172 & 10 & -0.651 & 0.394 & 4.11 & 0.162 & 0.635 & 5.21 \\
\hline 10 & 8 & 0.172 & 30 & -0.571 & 0.671 & 5.36 & 0.118 & 0.686 & 5.42 \\
\hline 10 & 8 & 0.172 & 100 & -0.165 & 2.28 & 9.87 & 0.0121 & 0.281 & 3.47 \\
\hline 10 & 8 & 0.172 & 300 & -0.0725 & 2.97 & 11.3 & 0.000450 & 3.92 & 13.0 \\
\hline 20 & 1 & 0.0272 & 1 & -0.696 & 0.0194 & 5.79 & 0.302 & 0.0463 & 8.94 \\
\hline 20 & 1 & 0.0272 & 3 & -0.704 & 0.0189 & 5.71 & 0.301 & 0.0481 & 9.12 \\
\hline 20 & 1 & 0.0272 & 10 & -0.739 & 0.0216 & 6.10 & 0.637 & 0.0525 & 9.52 \\
\hline 20 & 1 & 0.0272 & 30 & -0.336 & 0.0579 & 10.0 & 0.271 & 0.106 & 13.5 \\
\hline 20 & 1 & 0.0272 & 100 & -0.153 & 0.0358 & 7.86 & 0.113 & 0.0589 & 10.1 \\
\hline 20 & 1 & 0.0272 & 300 & -0.0924 & 0.0404 & 8.35 & 0.0716 & 0.0590 & 10.1 \\
\hline 20 & 2 & 0.0543 & 1 & -0.826 & 0.0889 & 6.20 & 0.790 & 0.201 & 9.33 \\
\hline 20 & 2 & 0.0543 & 3 & -0.814 & 0.0901 & 6.24 & 0.765 & 0.203 & 9.35 \\
\hline 20 & 2 & 0.0543 & 10 & -0.932 & 0.0758 & 5.72 & 1.37 & 0.213 & 9.58 \\
\hline 20 & 2 & 0.0543 & 30 & -0.535 & 0.129 & 7.47 & 0.824 & 0.233 & 10.0 \\
\hline 20 & 2 & 0.0543 & 100 & -0.234 & 0.141 & 7.80 & 0.285 & 0.256 & 10.5 \\
\hline 20 & 2 & 0.0543 & 300 & -0.227 & 0.0632 & 5.22 & 0.167 & 0.263 & 10.7 \\
\hline 20 & 4 & 0.109 & 1 & -0.835 & 0.436 & 6.86 & 0.601 & 0.778 & 9.16 \\
\hline 20 & 4 & 0.109 & 3 & -0.827 & 0.442 & 6.91 & 0.581 & 0.775 & 9.15 \\
\hline 20 & 4 & 0.109 & 10 & -0.966 & 0.327 & 5.94 & 1.13 & 0.835 & 9.49 \\
\hline 20 & 4 & 0.109 & 30 & -0.673 & 0.650 & 8.37 & 0.846 & 0.815 & 9.38 \\
\hline 20 & 4 & 0.109 & 100 & -0.284 & 0.734 & 8.90 & 0.256 & 0.894 & 9.82 \\
\hline 20 & 4 & 0.109 & 300 & -0.275 & 0.228 & 4.97 & 0.128 & 0.915 & 9.94 \\
\hline 20 & 8 & 0.217 & 1 & -0.727 & 1.74 & 6.86 & 0.208 & 1.88 & 7.13 \\
\hline 20 & 8 & 0.217 & 3 & -0.720 & 1.78 & 6.92 & 0.192 & 1.83 & 7.02 \\
\hline 20 & 8 & 0.217 & 10 & -0.882 & 1.09 & 5.41 & 0.433 & 2.41 & 8.06 \\
\hline 20 & 8 & 0.217 & 30 & -0.803 & 1.66 & 6.70 & 0.404 & 2.32 & 7.91 \\
\hline 20 & 8 & 0.217 & 100 & -0.290 & 3.36 & 9.52 & 0.105 & 2.11 & 7.55 \\
\hline 20 & 8 & 0.217 & 300 & -0.224 & 1.24 & 5.78 & 0.0384 & 1.92 & 7.20 \\
\hline 50 & 1 & 0.0369 & 1 & -0.790 & 0.0410 & 6.20 & 0.657 & 0.0743 & 8.35 \\
\hline 50 & 1 & 0.0369 & 3 & -0.797 & 0.0404 & 6.15 & 0.666 & 0.0775 & 8.52 \\
\hline 50 & 1 & 0.0369 & 10 & -0.819 & 0.0441 & 6.43 & 1.36 & 0.120 & 10.6 \\
\hline 50 & 1 & 0.0369 & 30 & -0.537 & 0.0394 & 6.07 & 0.571 & 0.102 & 9.80 \\
\hline 50 & 1 & 0.0369 & 100 & -0.209 & 0.0545 & 7.14 & 0.232 & 0.104 & 9.87 \\
\hline 50 & 1 & 0.0369 & 300 & -0.131 & 0.0582 & 7.39 & 0.149 & 0.105 & 9.94 \\
\hline 50 & 2 & 0.0737 & 1 & -0.914 & 0.196 & 6.78 & 1.64 & 0.276 & 8.04 \\
\hline 50 & 2 & 0.0737 & 3 & -0.910 & 0.196 & 6.77 & 1.60 & 0.281 & 8.12 \\
\hline 50 & 2 & 0.0737 & 10 & -0.957 & 0.189 & 6.66 & 3.27 & 0.267 & 7.92 \\
\hline 50 & 2 & 0.0737 & 30 & -0.731 & 0.204 & 6.91 & 1.77 & 0.332 & 8.83 \\
\hline 50 & 2 & 0.0737 & 100 & -0.333 & 0.220 & 7.18 & 0.557 & 0.403 & 9.72 \\
\hline 50 & 2 & 0.0737 & 300 & -0.211 & 0.229 & 7.32 & 0.328 & 0.428 & 10.0 \\
\hline 50 & 4 & 0.147 & 1 & -0.964 & 0.885 & 7.20 & 1.65 & 1.40 & 9.05 \\
\hline 50 & 4 & 0.147 & 3 & -0.963 & 0.883 & 7.19 & 1.62 & 1.42 & 9.12 \\
\hline 50 & 4 & 0.147 & 10 & -0.998 & 0.843 & 7.03 & 3.46 & 1.61 & 9.71 \\
\hline
\end{tabular}


Table 1

(Continued)

\begin{tabular}{|c|c|c|c|c|c|c|c|c|c|}
\hline $\begin{array}{l}m_{p} \\
\left(M_{\oplus}\right)\end{array}$ & $\begin{array}{c}a \\
(\mathrm{AU})\end{array}$ & $\begin{array}{l}r_{\text {Hill }} \\
(\mathrm{AU})\end{array}$ & $\begin{array}{c}\lambda \\
(\mu \mathrm{m})\end{array}$ & $\begin{array}{l}\text { Shadow } \\
\text { Contrast }\end{array}$ & $\begin{array}{c}\text { Area } \\
\left(\mathrm{AU}^{2}\right)\end{array}$ & $\frac{r_{\text {spot }}}{r_{\text {Hill }}}$ & $\begin{array}{c}\text { Bright } \\
\text { Contrast }\end{array}$ & $\begin{array}{c}\text { Area } \\
\left(\mathrm{AU}^{2}\right)\end{array}$ & $\frac{r_{\text {spot }}}{r_{\text {Hill }}}$ \\
\hline 50 & 4 & 0.147 & 30 & -0.864 & 1.02 & 7.71 & 2.57 & 1.57 & 9.58 \\
\hline 50 & 4 & 0.147 & 100 & -0.422 & 0.986 & 7.60 & 0.683 & 1.94 & 10.7 \\
\hline 50 & 4 & 0.147 & 300 & -0.253 & 0.997 & 7.64 & 0.345 & 2.03 & 10.9 \\
\hline 50 & 8 & 0.295 & 1 & -0.979 & 3.00 & 6.63 & 0.837 & 5.53 & 9.00 \\
\hline 50 & 8 & 0.295 & 3 & -0.971 & 3.04 & 6.68 & 0.810 & 5.53 & 9.00 \\
\hline 50 & 8 & 0.295 & 10 & -0.985 & 2.92 & 6.54 & 1.53 & 6.13 & 9.48 \\
\hline 50 & 8 & 0.295 & 30 & -0.942 & 3.51 & 7.17 & 1.60 & 5.98 & 9.36 \\
\hline 50 & 8 & 0.295 & 100 & -0.436 & 4.35 & 7.98 & 0.486 & 6.51 & 9.77 \\
\hline 50 & 8 & 0.295 & 300 & -0.274 & 3.42 & 7.08 & 0.202 & 6.77 & 9.96 \\
\hline
\end{tabular}

mission. However, there are no near-term prospects for such a mission.

In the radio, the Atacama Large Millimeter/submillimeter Array (ALMA) ${ }^{9}$ will have mas resolution when it reaches its full operating capacity in 2012. While the resolution would be good enough to resolve a planet shadow, the perturbations have very low contrast at $0.3 \mathrm{~mm}$, the shortest wavelength that ALMA will operate at. The problem here is one of sensitivity rather than angular resolution.

This study was restricted to planets forming interior to $10 \mathrm{AU}$, based on scenarios of giant planet formation within our own solar system. However, discoveries of planets around HR 8799 and Fomalhaut indicate that exoplanets can form tens to hundreds of AU from their stars (Marois et al. 2008; Kalas et al. 2008). The size of planet shadows in protoplanetary disks scale roughly with the planet-star distance. So there is hope that planets forming at these far distances from their stars might be observable in the near future, perhaps even with current instrumentation.

\section{CONCLUSIONS}

Observable perturbations from planets roughly scale with planet mass and orbital distance. The features are most easily observed in visible to mid-infrared wavelengths. The visible regime probes scattered light off the surface of the disk, revealing shadows caused by the gravitational potential of the planet. Mid-IR wavelengths reveal cooling/heating from shadowed/ brightened regions at the surface. Confirmation of shadows and brightenings seen in scattered light images of disks should therefore be carried out at near- to mid-IR wavelengths. In longer wavelengths toward the submillimeter, the contrast of the perturbations becomes smaller as the disk becomes optically thin.

The validity of the simulations presented in this study is limited by the fact that hydrodynamics are not included. This means that large-scale effects such as gap-clearing are not well modeled. If an annular gap does form, this density perturbation will be subjected to shadowing and illumination in the same way local dimples are, so radiative feedback on gaps should enhance these structures as well. Thus, the local planet shadows modeled in this paper represent lower limits on perturbations to disks that might be caused by embedded planet cores.

Another missing physical process is accretion onto the forming planet core. This process is difficult to capture even in high-resolution three-dimensional hydrodynamic simulations because of the extremely high dynamic range necessary to resolve the final capture of gas into the atmosphere of the growing planet. Heating from accretion onto the planet could alter the results of this study. Simulations by Hubickyj et al. (2005) indicate that growing planets accrete slowly over a long period of time, but once it crosses a threshold mass of roughly $10-20 M_{\oplus}$, it accretes gas rapidly over a short time period. Thus, accretional heating is likely to be only transitionally important. On the other hand, planets might grow so rapidly that 50 Earth mass planets might never be seen, being only a transitional stage on the way to becoming a Jovian-type planet.

The author thanks John Debes for helpful discussions and advice in developing the dust opacity model used in this paper, Lee Mundy and an anonymous referee for helpful comments, and Matthew Condell for assistance with software development. This work was performed under contract with the Jet Propulsion Laboratory (JPL) funded by NASA through the Michelson Fellowship Program. JPL is managed for NASA by the California Institute of Technology.

\section{REFERENCES}

Calvet, N., Patino, A., Magris, G. C., \& D’Alessio, P. 1991, ApJ, 380, 617 Chiang, E. I., \& Goldreich, P. 1997, ApJ, 490, 368

D'Alessio, P., Calvet, N., \& Hartmann, L. 2001, ApJ, 553, 321

D’Alessio, P., Calvet, N., Hartmann, L., Franco-Hernández, R., \& Servín, H. 2006, ApJ, 638, 314

D’Alessio, P., Calvet, N., Hartmann, L., Lizano, S., \& Cantó, J. 1999, ApJ, 527, 893

D’Alessio, P., Canto, J., Calvet, N., \& Lizano, S. 1998, ApJ, 500, 411

Dullemond, C. P., Dominik, C., \& Natta, A. 2001, ApJ, 560, 957

Eisner, J. A., Graham, J. R., Akeson, R. L., \& Najita, J. 2009, ApJ, 692, 309

Eisner, J. A., Hillenbrand, L. A., White, R. J., Akeson, R. L., \& Sargent, A. I. 2005, ApJ, 623, 952

Hartmann, L., Calvet, N., Gullbring, E., \& D’Alessio, P. 1998, ApJ, 495, 385

Hubickyj, O., Bodenheimer, P., \& Lissauer, J. J. 2005, Icarus, 179, 415

Isella, A., \& Natta, A. 2005, A\&A, 438, 899

Jang-Condell, H. 2008, ApJ, 679, 797

Jang-Condell, H., \& Boss, A. P. 2007, ApJ, 659, L169

Kalas, P., et al. 2008, Science, 322, 1345

Kenyon, S. J., Dobrzycka, D., \& Hartmann, L. 1994, AJ, 108, 1872

Klahr, H., \& Kley, W. 2006, A\&A, 445, 747

Marois, C., Macintosh, B., Barman, T., Zuckerman, B., Song, I., Patience, J., Lafrenière, D., \& Doyon, R. 2008, Science, 322, 1348

Muzerolle, J., Calvet, N., Hartmann, L., \& D’Alessio, P. 2003, ApJ, 597, L149

Pollack, J. B., \& Cuzzi, J. N. 1980, J. Atmos. Sci., 37, 868

Pollack, J. B., Hollenbach, D., Beckwith, S., Simonelli, D. P., Roush, T., \& Fong, W. 1994, ApJ, 421, 615

Shakura, N. I., \& Sunyaev, R. A. 1973, A\&A, 24, 337

Siess, L., Dufour, E., \& Forestini, M. 2000, A\&A, 358, 593

Wolf, S. 2008, Ap\&SS, 313, 109 